\title{
Jovens vivendo com HIV/AIDS: a influência da rede de relações, do coping e do neuroticismo sobre a satisfação de vida
}

\author{
Young people living with HIV/AIDS: the influence of relationship network, \\ coping and neuroticism on life satisfaction
}

\author{
Adriana Jung Serafini' ${ }^{1}$, Denise Ruschel Bandeira ${ }^{2}$
}

${ }^{1}$ Doutora em Psicologia, Universidade Federal do Rio Grande do Sul (UFRGS), Porto Alegre, RS. Professora, Departamento de Psicologia, Universidade Federal de Ciências da Saúde (UFCSPA). ${ }^{2}$ Doutora em Psicologia, UFRGS. Professora adjunta, Graduação e Pós-Graduação, Instituto de Psicologia, UFRGS. Programa de Pós-Graduação em Psicologia, Instituto de Psicologia, Universidade Federal do Rio Grande do Sul (UFRGS), Porto Alegre, RS. Conselho Nacional de Desenvolvimento Científico e Tecnológico (CNPq) e Coordenação de Aperfeiçoamento de Pessoal de Nível Superior (CAPES).

\section{Resumo}

Objetivos: Verificar o efeito das variáveis rede de relações, neuroticismo e estratégias de coping sobre a variável satisfação de vida em jovens soropositivos. Também buscou-se apresentar um perfil descritivo da amostra para as variáveis investigadas.

Método: Participaram 45 jovens soropositivos, com média de idade de 18,7 anos (desvio padrão =2,7), procedentes de três centros de atendimento da Grande Porto Alegre. Os instrumentos utilizados foram um questionário de dados sociodemográficos, a Escala Multidimensional de Satisfação de Vida para Adolescentes, o Inventário de Rede de Relações, o Inventário de Estratégias de Coping e a Escala Fatorial de Ajustamento Emocional/ Neuroticismo. Os dados dos instrumentos foram analisados através de análises de covariância e de variância multivariadas e análise de regressão múltipla.

Resultados: De forma geral, o nível de satisfação de vida dos jovens portadores do vírus HIV é bom, porém há um certo declínio na satisfação com as amizades. Em relação às estratégias de coping, as médias entre elas foram semelhantes. Níveis altos de neuroticismo foram evidenciados nos participantes, em especial no que se refere ao fator depressão. Esse foi o fator que melhor predisse a satisfação de vida total da amostra.

Conclusões: Pode-se perceber onde residem as maiores dificuldades desse grupo e, dessa forma, elaborar programas de intervenção efetivos.

Descritores: HIV, qualidade de vida, estresse, personalidade, relações interpessoais, adolescentes.

\begin{abstract}
Objectives: To investigate the effect of the variables network of relationships, neuroticism, and coping strategies on life satisfaction of HIV positive youths, and to present a descriptive profile of the sample for the studied variables.

Methods: Forty-five HIV positive youths from three health care centers located in the metropolitan area of Porto Alegre, whose mean age was 18.7 years (standard deviation $=2.7$ ), participated in this study. The following assessment instruments were used: a sociodemographic questionnaire, the Multidimensional Life Satisfaction Scale for Adolescents, the Network of Relationships Inventory, the Coping Strategies Inventory and the Neuroticism Factorial Scale. Data obtained with these instruments were analyzed using multivariate analysis of variance and covariance and linear multiple regression.

Results: Young HIV carriers present high life satisfaction, although there is a decrease in satisfaction with friendships. As far as coping strategies are concerned, the means between coping strategies were similar. High levels of neuroticism were evidenced in the sample, especially in terms of depression, which was the best predictor of global life satisfaction for the sample.

Conclusions: We were able to identify the main difficulties of this group of HIV carriers, and, based on these findings, it will be possible to develop effective intervention programs.
\end{abstract}

Keywords: HIV, quality of life, stress, personality, interpersonal relationships, adolescents.

\section{Correspondência:}

Adriana Jung Serafini, Instituto de Psicologia, UFRGS, Rua Ramiro Barcelos, 2600/120, CEP 90035-903, Porto Alegre, RS. Fax: (51) 3308.5470. E-mail: adrianaserafini@terra.com.br

Não há conflitos de interesse associados à publicação deste artigo.

Copyright (C) Revista de Psiquiatria do Rio Grande do Sul - APRS

Recebido em 02/10/2008. Aceito em 11/11/2008. 


\section{Introdução}

A AIDS tem sido caracterizada como uma epidemia mundial. De acordo com o Programa Conjunto das Nações Unidas sobre o HIV/AIDS ${ }^{1}$, ela é um fenômeno único na história da humanidade pela sua rápida propagação, alcance e impacto. Essa epidemia vem afetando desproporcionalmente os jovens de uma forma geral, sendo que pessoas entre 15 e 24 anos já representam a metade dos novos casos de HIV em todo o mundo. No Brasil, através dos últimos dados estatísticos divulgados pelo Boletim Epidemiológico - AIDS e DST ${ }^{2}$, no ano de 2007, 10,6\% das mulheres e 8,7\% dos homens diagnosticados como portadores do vírus HIV estavam na faixa dos 13 aos 24 anos de idade.

Muitos estudos sobre HIV, ao abordar a população adolescente, exploram questões importantes, como a prevenção do contágio do vírus ${ }^{3-5}$ ou os fatores de risco ou de proteção para o contágio do HIV ${ }^{6,7}$. Entretanto, poucas são as pesquisas que enfocam os adolescentes contaminados, em especial os seus aspectos psicológicos ${ }^{8-10}$. Estudos realizados no Brasil são ainda mais $\operatorname{raros}^{11-13}$. A presente pesquisa busca avaliar esses aspectos psicológicos, focando-se na avaliação da satisfação de vida de jovens portadores do vírus HIV. De acordo com Pavot et al. ${ }^{14}$, a satisfação de vida é considerada o elemento cognitivo que constitui o bem-estar subjetivo. Ela é definida como uma avaliação global que a pessoa faz de sua própria vida. Além disso, pesquisas indicam que certas variáveis podem influenciar o nível de bem-estar e, como consequência, o nível de satisfação. Entre essas variáveis estariam: traços de personalidade, eventos de vida e estratégias de coping e a presença de uma rede de suporte social e o estabelecimento de relações de intimidade ${ }^{15-18}$.

As pesquisas que têm como foco os portadores de HIV revelam que a forma como o indivíduo encara o diagnóstico depende da dinâmica psíquica anteriormente existente. Porém, de uma forma geral, as pessoas sentem o diagnóstico de infecção por HIV como um atestado de finitude e a limitação de desejos futuros. Surgem medos relacionados ao abandono, discriminação e perdas sociais, assim como sentimentos de culpa, raiva e pânico ${ }^{19,20}$.

Estudos que avaliam o nível de bem-estar ou satisfação de vida verificaram que ocorre uma diminuição no senso de bem-estar dos adolescentes soropositivos ${ }^{21}$. Problemas como estresse, discriminação e consequente diminuição do suporte social, depressão, ansiedade, dificuldade em revelar seu estado soropositivo para outras pessoas, assim como problemas relacionados ao desempenho escolar foram observados em adolescentes infectados pelo HIV ${ }^{8,22-24}$.

Conforme Wiener \& Septimus ${ }^{24}$, os maiores problemas que a infecção por HIV pode causar aos adolescentes emergem no momento de estabelecer relações fora da família. As grandes dificuldades se encontram em situações tais como informar sua condição ao parceiro sexual, a prática de sexo seguro, o medo das conclusões que seus pares podem ter sobre como foi infectado, culpa, sentir-se sujo ou sexualmente anormal. Reitera-se, por isso, a importância de avaliar as relações da pessoa infectada com seus amigos e familiares e o espaço existente para falar sobre seus sentimentos e angústias.

Percebe-se que o diagnóstico de HIV positivo durante uma fase já conflituosa por si só gera muitas angústias na vida de um adolescente. Uma das formas de entender como os adolescentes soropositivos lidam com os problemas oriundos de seu diagnóstico seria através da investigação das estratégias de coping utilizadas. Coping é definido por Lazarus \& Folkman ${ }^{25}$ como os esforços cognitivos e comportamentais para lidar com demandas externas e/ou internas específicas que são avaliadas como excedendo os esforços e recursos do indivíduo. A importância em se estudar as estratégias de coping está no fato de que sua efetividade relaciona-se com resultados positivos sobre aspectos da saúde física ou mental das pessoas. Assim, pode-se também relacionar tal efetividade à satisfação de vida ${ }^{15}$.

Meijer et al. ${ }^{26}$, em uma pesquisa com adolescentes portadores de doenças crônicas, afirmaram que as estratégias de coping que se mostraram como determinantes importantes para um funcionamento psicossocial positivo foram as estratégias ativas para a solução de problemas. Além disso, as estratégias de coping que buscavam suporte social também se mostraram efetivas, mas apenas quando estavam relacionadas a habilidades sociais. Desse modo, percebe-se uma relação entre as variáveis vinculadas a rede de relações (suporte social/intimidade) e estilos de coping que pode resultar em um aumento ou decréscimo no nível de satisfação de vida.

Assim, o objetivo geral desta pesquisa foi verificar se existiria efeito da rede de relações, neuroticismo e coping (variáveis independentes) sobre a satisfação de vida dos adolescentes soropositivos (variável dependente). Como objetivo específico, buscou-se apresentar um perfil descritivo da amostra para todas as variáveis citadas.

\section{Método}

\section{Amostra}

Participaram deste estudo um total de 45 jovens portadores do vírus HIV, com idade entre 14 e 23 anos [média $=18,7$; desvio padrão $(\mathrm{DP})=2,7]$, sendo $64,4 \%$ do sexo feminino e procedentes de três centros de atendimento da Grande Porto Alegre. Desse número total, 82,2\% dos participantes se encontravam em atendimento no Serviço de Atenção Terapêutica do Hospital Sanatório Partenon, 15,6\% no Centro de Testagem e Aconselhamento (CTA) de Viamão e 2,2\% no CTA do Ambulatório de Dermatologia. Em relação aos critérios de exclusão, não foram incluídos no estudo jovens não alfabetizados ou institucionalizados. Fizeram parte da amostra todos os pacientes dos centros de atendimento que possuíam a idade dentro do intervalo 
estabelecido e que aceitaram participar da pesquisa durante o período reservado para a coleta de dados.

Um maior percentual de jovens $(60 \%)$ possuía escolaridade entre $5^{\mathrm{a}}$ e $8^{\mathrm{a}}$ série do ensino fundamental e pertenciam à classe social $\mathrm{C}(55,6 \%)$, cuja renda média é de aproximadamente $\mathrm{R} \$ 927,00^{27}$. No que se refere ao estado civil, o percentual mais alto foi para os participantes solteiros (55,6\% do grupo clínico), seguido por aqueles que viviam com companheiro $(31,1 \%)$. Os dados coletados relativos ao HIV demonstram que a maior causa de infecção para esse grupo foi a prática de relação sexual desprotegida $(68,9 \%)$ e que, dos 45 jovens participantes, 18 (40\%) haviam recebido diagnóstico de AIDS.

\section{Instrumentos}

Em todos os participantes foram aplicados os seguintes instrumentos (em ordem de aplicação):

- Questionário de dados sociodemográficos incluindo questões acerca da contaminação pelo vírus. A classificação da classe social foi obtida através do Critério Padrão de Classificação Econômica Brasii ${ }^{27}$.

- A Escala Multidimensional de Satisfação de Vida para Adolescentes (EMSV-A) de Arteche ${ }^{28}$, que consiste em uma adaptação da escala de Giacomoni \& Hutz $^{29}$ desenvolvida para crianças. Ela avalia satisfação de vida através de sete fatores (self, self-comparado, família, escola, não-violência, amizade e global). Seus 57 itens são respondidos através de uma escala likert com as seguintes opções de repostas: (1) nem um pouco, (2) bem pouco, (3) mais ou menos, (4) bastante e (5) muitíssimo.

- Inventário de Rede de Relações (IRR), de Furman $\&$ Buhrmester $^{30}$, adaptado para o Brasil por Marques $\& \mathrm{Horn}^{31}$. O inventário avalia 11 dimensões de relacionamentos interpessoais através de 33 itens. As dimensões englobadas pelo instrumento são: conflito, poder relativo, punição, satisfação e sete dimensões de apoio social (companhia, auxílio instrumental, autorrevelação, cuidado com o outro, afeição, valorização e aliança confiável). Essas dimensões foram classificadas com cada uma das seguintes pessoas: mãe, pai, melhor amigo(a), namorado(a)/marido (esposa)/ companheiro(a). As respostas são registradas em uma escala likert de cinco pontos, que indica a qualidade de relacionamento com cada uma das pessoas citadas.

- O Inventário de Estratégias de Coping de Folkman $\&$ Lazarus $^{32}$ adaptado para o português por Savóia et al. ${ }^{33}$, que avalia estratégias de coping através de oito fatores (confronto, afastamento, autocontrole, suporte social, aceitação de responsabilidade, fuga-esquiva, resolução de problemas e reavaliação positiva). Seus 66 itens são respondidos pelo participante de acordo com a intensidade com que utilizaram cada estratégia mencionada, em determinada situação, tendo como opções de respostas: (0) não usei esta estratégia, (1) usei um pouco, (2) usei bastante e (3) usei em grande quantidade.

- A Escala Fatorial de Ajustamento Emocional/Neuroticismo $(\mathrm{EFN})^{34}$, um instrumento que avalia a dimensão da personalidade humana denominada neuroticismo/estabilidade emocional (modelo dos Cinco Grandes Fatores) através do levantamento de traços de personalidade. O teste é composto por 82 itens em quatro subescalas (Escala de Vulnerabilidade, Escala de Desajustamento Psicossocial, Escala de Ansiedade e Escala de Depressão). Os itens são registrados em uma escala likert de sete pontos, em que $1=$ completamente inadequada, a sentença não descreve nenhuma característica minha, $4=$ neutro, mais ou menos e $7=$ perfeitamente adequada, a sentença me descreve perfeitamente bem.

\section{Procedimentos}

Como etapa inicial da coleta de dados, foram realizados contatos com três centros de atendimento da Grande Porto Alegre. Após aprovação do projeto pelos locais de coleta, foram solicitadas a todos os locais as listas de pacientes portadores do vírus HIV com idades entre 14 e 23 anos.

A partir da lista, os pacientes foram recrutados através de convite nos dias em que possuíam atendimento com os profissionais dos centros, ou através de busca ativa em que era marcada uma data para a realização da entrevista. A busca ativa somente não foi autorizada no CTA do Ambulatório de Dermatologia, pois não era prática utilizada no centro. A aplicação dos questionários foi realizada individualmente, em uma sala cedida pelo centro de atendimento.

\section{Análise dos dados}

Para fins de análise dos dados, foi utilizado o pacote estatístico Statistical Package for the Social Sciences (SPSS) versão 13.0. Na construção do banco de dados, as variáveis referentes às respostas aos instrumentos EMSVA, IRR, Inventário de Estratégias de Coping e EFN, que haviam sido deixadas em branco, foram substituídas pela média do grupo naquele item.

Inicialmente, os instrumentos foram avaliados do ponto de vista psicométrico, e os dados obtidos foram analisados através de estatísticas descritivas, sendo apresentadas médias e DP das diversas medidas. Para responder aos objetivos específicos, os dados dos instrumentos EMSV-A, IRR e EFN foram analisados através de análise de covariância multivariada (MANCOVA), sendo incluído o sexo como variável independente. Pelo fato de a variável idade ter se correlacionado com esses instrumentos, ela foi utilizada como controle nas análises. Como não foi encontrada diferença entre sexos nas análises do Inventário de Estratégias de Coping, realizou-se análise de variância multivariada 
(MANOVA), sendo a variável independente o tipo de situação de estresse experenciado.

Por fim, para responder ao objetivo geral deste estudo, foi realizada uma análise de regressão múltipla, para avaliar o efeito das variáveis independentes sobre a variável dependente (fatores relacionados à satisfação de vida). Nessa análise, foram consideradas como variáveis independentes os fatores do EFN, do Inventário de Estratégias de Coping e do IRR que se correlacionaram com o fator de satisfação de vida total.

\section{Aspectos éticos}

O projeto desta pesquisa foi aprovado pelo Comitê de Ética em Pesquisa da Escola de Saúde Pública do RS (CEPS/ESO - 091/2006). Antes de iniciar a aplicação dos instrumentos, os pacientes com idades a partir de 18 anos que aceitaram participar preencheram o termo de consentimento livre e esclarecido. Para os jovens com idade inferior a 18 anos que manifestaram interesse em respon- der à pesquisa, foi solicitado que o paciente e os pais ou responsáveis preenchessem o termo. Em ambos os casos o documento foi assinado em duas vias, uma permanecendo com a pesquisadora e a outra com o participante.

\section{Resultados}

\section{Escala Multidimensional de Satisfação de Vida para Adolescentes (EMSV-A)}

Em relação aos seus aspectos psicométricos, a EMSVA apresentou uma boa consistência interna $(\alpha=0,94)$. A Tabela 1 apresenta os resultados das análises das médias por sexo e faixa etária para todos os fatores da escala e sua soma total. Salienta-se que foram realizadas ANOVAS, sendo as variáveis dependentes os fatores da EMSV-A e a independente tempo de diagnóstico e, posteriormente, a presença de diagnóstico de AIDS, embora não tenham sido encontradas diferenças significativas.

Tabela 1 - Resultados da MANCOVA para os fatores da EMSV-A

\begin{tabular}{lcccc}
\hline Fatores & $\begin{array}{c}\text { Total }(\mathbf{n}=\mathbf{4 5}) \\
\text { Média (DP) }\end{array}$ & $\begin{array}{c}\text { Feminino (n= 29) } \\
\text { Média (DP) }\end{array}$ & $\begin{array}{c}\text { Masculino (n= 16) } \\
\text { Média (DP) }\end{array}$ & F \\
\hline Família & $4,07(0,69)$ & $3,95(0,71)$ & $4,28(0,62)$ & 0,60 \\
Self-comparado & $3,51(0,77)$ & $3,62(0,77)$ & $3,31(0,74)$ & 2,28 \\
Escola & $3,75(0,76)$ & $3,74(0,77)$ & $3,76(0,78)$ & 0,04 \\
Não-violência & $3,87(0,72)$ & $3,83(0,77)$ & $3,92(0,63)$ & 0,00 \\
Amizade & $3,63(0,79)$ & $3,47(0,79)$ & $3,92(0,74)$ & 1,61 \\
Self & $3,87(0,81)$ & $3,63(0,86)$ & $4,31(0,50)$ & $5,05^{*}$ \\
Satisfação de vida global & $3,72(0,95)$ & $3,45(0,99)$ & $4,21(0,65)$ & $4,03^{*}$ \\
Satisfação de vida total & $3,77(0,57)$ & $3,67(0,62)$ & $3,97(0,42)$ & 1,03 \\
\hline
\end{tabular}

$\mathrm{DP}=$ desvio padrão; EMSV-A = Escala Multidimensional de Satisfação de Vida para Adolescentes;

$\mathrm{F}=$ resultado da MANCOVA; MANCOVA = análise de covariância multivariada.

* Diferença significativa entre médias, $\mathrm{p} \leq 0,05$.

Através dos resultados da MANCOVA, percebe-se que os jovens da amostra, de forma geral, apresentaram bom nível de satisfação de vida, pois as médias dos fatores da EMSV-A variaram entre 3,51 e 4,07. Observou-se algumas diferenças entre os sexos, sendo que os jovens do sexo masculino apresentaram uma média significativamente maior nos fatores self e satisfação de vida global do que as jovens.

\section{Inventário de Rede de Relações}

O IRR também apresentou uma boa consistência interna. Essa análise foi realizada separadamente para cada uma das pessoas classificadas: mãe $(\alpha=0,90)$, pai $(\alpha=0,89)$, melhor amigo $(\alpha=0,93)$ e companheiro(a) $(\alpha=0,89)$.

Também foram desenvolvidas análises de covariância para os fatores que compõem o IRR. Elas foram realizadas individualmente para cada um dos relacionamentos citados anteriormente, tendo o sexo como variável independente.
Na Tabela 2, em função da grande quantidade de fatores, são apresentados somente os resultados cujas médias diferenciam-se entre os sexos.

Através dos resultados da MANCOVA, observou-se que os únicos fatores que apresentaram diferença entre os sexos foram aqueles vinculados às relações estabelecidas entre os jovens e as figuras paternas e entre os jovens e seus melhores amigos. As participantes do sexo feminino apresentaram médias significativamente mais altas nos fatores ligados à relação com o pai (satisfação na relação com o pai, relação de cuidado com o pai, afeição pelo pai, valorização pelo pai e aliança confiável com o pai), com exceção apenas do fator conflito com o pai, cuja média foi mais alta para os jovens do sexo masculino. Os participantes do sexo masculino, por sua vez, apresentaram médias significativamente mais altas nos fatores ligados à relação com o melhor amigo (satisfação na relação com o melhor amigo, afeição pelo melhor amigo e punição pelo melhor amigo). 
Tabela 2 - Resultados da MANCOVA para os fatores do IRR

\begin{tabular}{lccccccc}
\hline & \multicolumn{3}{c}{ Total } & \multicolumn{2}{c}{ Feminino } & \multicolumn{3}{c}{ Masculino } \\
\cline { 2 - 6 } Fatores & $\mathbf{n}$ & Média (DP) & n & Média (DP) & n & Média (DP) & F \\
\hline Conflito com o pai & 24 & $2,00(0,85)$ & 17 & $1,74(0,71)$ & 07 & $2,61(0,91)$ & $22,36^{*}$ \\
Satisfação na relação com o pai & 24 & $3,68(1,21)$ & 17 & $3,94(1,09)$ & 07 & $3,04(1,35)$ & $24,15^{*}$ \\
Relação de cuidado com o pai & 24 & $3,31(1,09)$ & 17 & $3,41(1,07)$ & 07 & $3,09(1,19)$ & $4,86^{\dagger}$ \\
Afeição pelo pai & 24 & $4,23(0,95)$ & 17 & $4,37(0,84)$ & 07 & $3,90(1,18)$ & $4,14^{\dagger}$ \\
Valorização pelo pai & 24 & $3,36(1,06)$ & 17 & $3,56(1,05)$ & 07 & $2,85(0,97)$ & $25,54^{*}$ \\
Aliança confiável com o pai & 24 & $4,12(1,04)$ & 17 & $4,21(0,99)$ & 07 & $3,90(1,21)$ & $5,44^{\dagger}$ \\
Satisfação na relação com o melhor amigo & 38 & $3,65(0,91)$ & 25 & $3,37(0,91)$ & 13 & $4,20(0,64)$ & $11,66^{*}$ \\
Afeição pelo melhor amigo & 38 & $3,45(0,94)$ & 25 & $3,17(0,88)$ & 13 & $4,00(0,84)$ & $4,87^{\dagger}$ \\
Punição pelo melhor amigo & 38 & $1,70(0,61)$ & 25 & $1,44(0,36)$ & 13 & $2,20(0,68)$ & $7,76^{\dagger}$ \\
\hline
\end{tabular}

$\mathrm{DP}=$ desvio padrão; $\mathrm{F}=$ resultado da MANCOVA; IRR = Inventário de Rede de Relações; MANCOVA = análise de covariância multivariada.

$*$ Diferença significativa entre médias, $\mathrm{p} \leq 0,01$.

${ }^{\dagger}$ Diferença significativa entre médias, $\mathrm{p} \leq 0,05$.

\section{Inventário de Estratégias de Coping}

O Inventário de Estratégias de Coping apresentou boa consistência interna $(\alpha=0,90)$. As análises de variância foram realizadas utilizando-se os oito fatores do instrumento como variáveis dependentes e a situação de estresse como fator fixo. As situações de estresse foram classificadas dentro de duas categorias. A primeira foi relacionada ao HIV, que incluiu situações como a descoberta do diagnóstico de HIV positivo, descobrir que o filho foi contaminado por transmissão vertical ou ficar muito doente/ser internado por complicações da infecção, a segunda foi vinculada a situações diversas não relacionadas ao HIV. Os resultados para todos os fatores são apresentados na Tabela 3 .

Os resultados demonstram que as estratégias mais utilizadas pelos participantes foram as classificadas no fator reavaliação positiva. Entretanto, as médias de todos os fatores foram bastante semelhantes, variando entre 1,09 e 1,53. Houve diferenças significativas entre as médias dos jovens que relataram a situação de estresse relacionada ao HIV e daqueles que descreveram outras situações. Enquanto o primeiro grupo utilizou mais as estratégias de autocontrole, aceitação de responsabilidade, fuga-esquiva e reavaliação positiva, o segundo fez mais uso das estratégias de confronto.

\section{Escala Fatorial de Ajustamento Emocional/Neuroti- cismo (EFN)}

A EFN apresentou boa consistência interna, com Alpha de Cronbach de 0,95. Os quatro fatores da escala foram utilizados na análise de covariância, e seus resultados são demonstrados na Tabela 4.

Tabela 3 - Resultados da MANOVA para os fatores do Inventário de Estratégias de Coping

\begin{tabular}{lcccc}
\hline & \multicolumn{3}{c}{ Situação de estresse } \\
\cline { 2 - 4 } Fatores & $\begin{array}{c}\text { Total (n= 45) } \\
\text { Média (DP) }\end{array}$ & $\begin{array}{c}\text { HIV (n = 20) } \\
\text { Média (DP) }\end{array}$ & $\begin{array}{c}\text { Não-HIV (n= 25) } \\
\text { Média (DP) }\end{array}$ & F \\
\hline Reavaliação positiva & $1,53(0,67)$ & $1,81(0,75)$ & $1,30(0,52)$ & $7,00^{\dagger}$ \\
Suporte social & $1,46(0,71)$ & $1,62(0,84)$ & $1,34(0,57)$ & 1,73 \\
Aceitação de responsabilidade & $1,38(0,79)$ & $1,74(0,78)$ & $1,10(0,70)$ & $8,42^{\dagger}$ \\
Autocontrole & $1,34(0,65)$ & $1,58(0,74)$ & $1,14(0,49)$ & $5,90^{*}$ \\
Afastamento & $1,24(0,75)$ & $1,43(0,80)$ & $1,09(0,68)$ & 2,35 \\
Fuga e esquiva & $1,22(0,60)$ & $1,41(0,62)$ & $1,06(0,56)$ & $4,02^{*}$ \\
Confronto & $1,09(0,50)$ & $0,92(0,49)$ & $1,23(0,47)$ & $4,44^{*}$ \\
Resolução de problemas & $1,09(0,65)$ & $1,24(0,74)$ & $0,97(0,55)$ & 2,04 \\
\hline
\end{tabular}

$\mathrm{DP}=$ desvio padrão $\mathrm{F}=$ resultado da MANOVA; MANOVA = análise de variância multivariada .

* Diferença significativa entre médias, $\mathrm{p} \leq 0,05$.

${ }^{\dagger}$ Diferença significativa entre médias, $\mathrm{p} \leq 0,01$. 
Tabela 4 - Resultados da MANCOVA para os fatores da EFN

\begin{tabular}{lccc}
\hline & \multicolumn{3}{c}{ Sexo } \\
\cline { 2 - 3 } Fatores & Feminino (n= 29) & Masculino (n= 16) & \\
\hline Vulnerabilidade & Média (DP) & Média (DP) & F \\
Ansiedade & $80,72(25,67)$ & $72,56(23,06)$ & 0,07 \\
Desajustamento & $92,82(33,13)$ & $85,56(28,27)$ & 0,01 \\
Depressão & $23,55(8,26)$ & $32,12(16,68)$ & $7,70^{*}$ \\
\hline
\end{tabular}

$\mathrm{DP}=$ desvio padrão EFN = Escala Fatorial de Ajustamento Emocional/Neuroticismo;

$\mathrm{F}=$ resultado da MANCOVA; MANCOVA = análise de covariância multivariada.

* Diferença significativa entre médias, $\mathrm{p} \leq 0,01$.

Os resultados demonstram diferença significativa entre os sexos apenas no fator desajustamento. Nesse fator, os jovens do sexo masculino apresentaram uma média mais alta do que as jovens.

\section{Análise de regressão linear multivariada}

Como o número de sujeitos desta amostra era reduzido, para a análise optou-se por utilizar um fator maior do IRR, o suporte social que é formado pelas dimensões companhia, auxílio instrumental, revelação/intimidade, cuidado com o outro, afeição, valorização e aliança confiável, conforme indicado por Marques \& Horn $^{31}$. Foram realizadas análises de correlação entre sexo, idade, os fatores de todas as escalas (IRR, Inven- tário de Estratégias de Coping e EFN) e o fator satisfação de vida total da EMSV-A. Os fatores que correlacionaram foram Idade, vulnerabilidade (EFN), ansiedade (EFN), depressão (EFN), confronto (coping), fuga-esquiva (coping), apoio social da mãe (IRR), apoio social do melhor amigo (IRR), satisfação com o pai (IRR) e satisfação com a mãe (IRR). Como os dois últimos fatores também avaliam satisfação, como a EMSV-A, eles não foram incluídos na análise de regressão linear multivariada. Assim, para esta análise, foram utilizadas como variáveis independentes idade e os fatores depressão, ansiedade e vulnerabilidade do EFN, confronto e fuga-esquiva do Inventário de Estratégias de Coping e apoio social da mãe e apoio social do melhor amigo do IRR. Na Tabela 5 podem ser observados os resultados da análise.

Tabela 5 - Resultados da análise de regressão linear multivariada

\begin{tabular}{lcccc}
\hline & \multicolumn{4}{c}{ Satisfação de vida total } \\
\cline { 2 - 5 } Variáveis independentes & $\beta$ & $\mathbf{R}$ & $\mathbf{R}^{2}$ & $\mathbf{R}^{2}$ ajustado \\
\hline Depressão & $-0,62$ & 0,78 & 0,61 & 0,60 \\
Apoio social da mãe & 0,30 & 0,83 & 0,69 & 0,67 \\
Confronto & $-0,22$ & 0,85 & 0,73 & 0,70 \\
\hline$\beta=$ valor padronizado da inclinação da linha de regressão; $\mathrm{R}=$ correlação entre todas as variáveis preditoras com \\
a variável de critério; $\mathrm{R}^{2}=$ variância explicada pelo modelo; $\mathrm{R}^{2}$ ajustado $=\mathrm{R}^{2}$ ajustado em relação à amostra.
\end{tabular}

Os resultados da análise de regressão linear multivariada indicam que os fatores depressão (EFN), apoio social da mãe (IRR) e confronto (Inventário de Estratégias de Coping) reunidos compreendem $70 \%$ da variância da satisfação de vida total dos jovens da amostra. Somente o fator depressão é responsável por $60 \%$ dessa variância.

\section{Discussão e conclusões}

Os resultados da presente pesquisa demonstraram que os participantes apresentaram bom nível de satisfação de vida.
Esses resultados vão de encontro a estudos que demonstram que adolescentes e/ou jovens soropositivos tendem a ter um rebaixamento no nível de bem-estar ou satisfação de vida ${ }^{8,21}$. Em razão de tais achados, procurou-se investigar que aspectos poderiam estar atuando como promotores da satisfação de vida desse grupo investigado. Assim, foram avaliadas as características das relações estabelecidas pelos jovens portadores do HIV, as situações de estresse enfrentadas por eles e as estratégias de coping associadas e também neuroticismo. Uma análise de regressão múltipla foi realizada com o objetivo de determinar quais variáveis foram, efetivamente, preditoras da satisfação de vida desse grupo. 
Ainda no que se refere à análise dos resultados da EMSV-A, pode-se observar um nível de satisfação de vida alto vinculado à dimensão família. Observa-se que tal dimensão obteve a média mais alta dentre todas as dimensões para o grupo total $(4,07, \mathrm{DP}=0,69)$, e que não houve diferença significativa entre os sexos. Essa média significa que os jovens da amostra encontravam-se bastante satisfeitos nos aspectos de suas vidas relacionados à família. Também em relação à influência da família sobre os jovens da amostra, através da análise de regressão múltipla, pôde-se constatar que o fator apoio social da mãe, do IRR, foi um dos responsáveis na predição da satisfação de vida dos jovens participantes. Esses resultados indicam que, assim como já havia sido apontado em estudos anteriores ${ }^{21}$, o suporte social dos pais é um componente importante para a promoção de aspectos relacionados ao bem-estar em jovens soropositivos.

Ao mesmo tempo em que a família aparece com a média mais alta dentre as dimensões da EMSV-A, percebe-se que as dimensões self-comparado (exemplo de item: "os outros jovens são mais alegres do que eu") e amizade possuem as médias mais baixas (média $=3,51, \mathrm{DP}=0,77$; média $=$ $3,63, \mathrm{DP}=0,79$, respectivamente). Apesar de essas médias ainda ficarem próximas da classificação 4 , que indica que os jovens estão bastante satisfeitos com essas áreas de suas vidas, pode-se pensar que, para os jovens soropositivos, há certo declínio na satisfação relacionada à amizade. Wiener $\&$ Septimus $^{24}$ referem que dentre os maiores problemas que a infecção pelo HIV pode causar na vida dos jovens está no momento de estabelecer relações fora da família, pelo medo das conclusões que seus pares podem chegar sobre a forma da contaminação e pela culpa sentida. Tais questões podem estar interferindo na qualidade da relação que esses jovens estabelecem com seus pares, mas mesmo assim, na análise de regressão linear múltipla, o fator suporte social de amigos não chegou a predizer o nível de satisfação de vida desse grupo.

Além da presença de um dos fatores do IRR como variável preditora na análise de regressão, pode-se observar algumas diferenças entre os jovens dos sexos feminino e masculino da amostra através da MANCOVA realizada para análise de todos os fatores do instrumento. Observou-se que as participantes do sexo feminino apresentaram médias significativamente mais altas nos fatores ligados à relação com o pai. Os fatores que pertencem a um fator maior, o de suporte social, foram os que mais diferenciaram esse grupo, englobando afeição pelo pai, valorização pelo pai e aliança confiável com o pai. As jovens também apresentaram médias mais altas para os fatores satisfação na relação com o pai e relação de cuidado com o pai. Os jovens do sexo masculino, por sua vez, apresentaram médias mais altas para o fator conflito com o pai. Eles também apresentaram médias significativamente mais altas nos fatores ligados a relação com o melhor amigo (satisfação na relação com o melhor amigo, afeição pelo melhor amigo e punição pelo melhor amigo). Isso parece demonstrar que enquanto para as jovens o pai parece ser uma figura que oferece um suporte positivo, para os participantes do sexo masculino as relações conflituosas com ele são mais presentes. Já as relações de amizade e suas características tanto positivas (satisfação e afeição) quanto negativas (punição) aparecem mais fortes para o grupo masculino. Tais resultados diferenciam-se dos obtidos com jovens não-portadores do HIV, que caracterizam as relações de jovens do sexo feminino com seus amigos como mais profundas e afetivas ${ }^{35,36}$.

Outros fatores que interferem no nível de bem-estar e satisfação de vida dos portadores do HIV são os eventos estressores e as estratégias de coping utilizadas para lidar com eles ${ }^{8,22}$. No que se refere às estratégias utilizadas pelos jovens para lidar com problemas, de uma forma geral, não se observou grande variação na média dos fatores. Pôde-se perceber que o único fator cuja média aproximou-se da classificação 2 foi o de reavaliação positiva (média $=1,53$, $\mathrm{DP}=0,67)$. Essa classificação significa que o participante utilizou "bastante" as estratégias em questão. Para todos os demais fatores, as médias totais aproximaram-se da classificação 1, que corresponde a terem utilizado "um pouco" as estratégias. Observou-se, também, que o grupo de participantes que mais fez uso da reavaliação positiva foi o dos jovens que relacionaram as estratégias a uma situação vinculada ao HIV.

Houve diferenças significativas entre as médias dos jovens que relataram a situação de estresse relacionada ao HIV e daqueles que descreveram outras situações. Enquanto o primeiro grupo utilizou mais as estratégias de autocontrole, aceitação de responsabilidade, fuga-esquiva e reavaliação positiva, o segundo fez mais uso das estratégias de confronto. Apesar de a média do fator confronto ter sido a única significativamente mais alta para o grupo que não relacionou a situação de estresse ao HIV, a média mais alta de estratégias utilizadas foi para os fatores suporte social e reavaliação positiva. É interessante observar que, na análise de regressão múltipla, o único fator do Inventário de Estratégias de Coping que predisse a satisfação de vida da amostra geral foi o confronto, sendo que a diminuição do uso de tais estratégias acarretava em aumento da satisfação de vida. Levando-se em conta que neste estudo foi utilizada a diretriz de avaliação da efetividade das estratégias de coping proposta por Aldwin \& Reverson ${ }^{15}$ que relaciona coping efetivo com aspectos relacionados à saúde física $\mathrm{e}$ mental, poderíamos compreender que, para os jovens desta pesquisa, o uso de estratégias de confronto não seria efetivo para a aquisição da satisfação de vida.

Pelo fato de o grupo de jovens que relatou a situação de estresse relacionada ao HIV ter utilizado as estratégias de confronto em uma média significativamente mais baixa do que os demais, pode-se levantar a hipótese de que aqueles jovens possivelmente percebem a infecção como uma situação estressante, mas que pode ser enfrentada. Provavelmente, o fato de conseguirem compreender que a 
infecção pelo vírus é um acontecimento grave, vai fazer com que tais jovens se preocupem com sua saúde e busquem o atendimento adequado. Além de apresentarem uma média mais baixa no fator confronto, esses jovens apresentaram uma média significativamente mais alta no fator reavaliação positiva. Apesar deste último não ter sido uma das variáveis que predisse a satisfação de vida do grupo, sua utilização pode ter um caráter positivo sobre a vida desses participantes, já que significa que a pessoa realizou uma reflexão acerca da situação vivenciada e pode obter dela algum aprendizado (exemplo de item do fator reavaliação positiva: "mudei ou cresci como pessoa de uma maneira positiva"). Percebe-se também que esse mesmo grupo apresentou médias significativamente mais altas em outros fatores de estratégias, demonstrando, assim, que os jovens que associaram a situação de estresse ao HIV apresentam um repertório maior e maior intensidade na utilização de estratégias de coping.

Em relação ao EFN, observou-se que as médias dos jovens desta amostra apresentaram-se dentro de percentis considerados como médios, quando comparadas com a amostra padronizada do instrumento ${ }^{34}$, apenas nos fatores vulnerabilidade (percentil 60) para o sexo masculino e desajustamento (percentil 50) para o sexo feminino. Neste último fator, inclusive, os participantes do sexo masculino apresentaram uma média significativamente mais alta do que as do sexo feminino, sendo aquela classificada como um percentil alto (percentil 70). Os jovens do sexo masculino ainda apresentaram percentis altos para os fatores ansiedade (percentil 75) e depressão (percentil 65). Já as jovens obtiveram percentis altos nos fatores vulnerabilidade (percentil 65), ansiedade (percentil 75) e muito alto no fator depressão (percentil 85).

De acordo com o manual do $\mathrm{EFN}^{34}$, resultados altos no fator vulnerabilidade sugerem indivíduos com características de insegurança e baixa autoestima, que se preocupam muito em serem aceitos pelos outros e que sofrem quando isso não ocorre. Já o fator desajustamento se refere a características de agressividade e hostilidade, assim como a comportamentos de risco, como os sexuais, abuso de álcool e drogas e envolvimento com jogos de azar.

Apesar de não ter ocorrido diferença significativa entre os sexos no fator vulnerabilidade, pela interpretação dos escores médios dos grupos poderíamos dizer que ele está caracterizando mais o grupo de jovens do sexo feminino desta amostra do que os do sexo masculino. Já os homens apresentariam mais características relacionadas ao fator desajustamento.

A partir desses resultados, poderíamos inferir que as características de personalidade das jovens portadoras do vírus HIV, como insegurança e necessidade de serem aceitas pelos outros (muitas vezes o parceiro), que são evidenciadas pela média alta no fator vulnerabilidade, acabam as expondo ao risco da contaminação. Para os homens, por sua vez, características como as de comportamento de risco, perce- bidos pela média alta no fator desajustamento, têm atuado como fator de exposição à infecção. Resultados de pesquisas anteriores suportam essas interpretações, relacionando-as especialmente a características específicas de gênero e cultura $^{37,38}$. De acordo com elas, jovens do sexo feminino tendem a privilegiar as relações afetivas, submetendo-se aos parceiros quando esses se recusam a utilizar preservativo nas relações sexuais, até como uma forma de afirmar sua fidelidade e confiança no parceiro, colocando em risco sua própria saúde. Já os homens, apesar de utilizarem mais o preservativo, tendem a se colocar em mais situações perigosas, como o uso de drogas, início da atividade sexual mais precoce e com número maior de parceiros.

$\mathrm{Na}$ análise de regressão linear múltipla, a variável que melhor predisse a satisfação de vida dos participantes foi o fator depressão (a ausência de). Percebe-se, dessa forma, que aspectos da personalidade que caracterizam o fator depressão do EFN, como sentimentos de solidão, baixa expectativa quanto ao futuro e falta de objetivos na $v \operatorname{lida}^{34}$, são determinantes para rebaixamento do nível de satisfação de vida dos jovens.

Os resultados também indicaram que os jovens soropositivos desta amostra, de ambos os sexos, apresentaram médias altas para os fatores depressão e ansiedade. Sintomas de ansiedade e depressão já foram encontrados em estudos prévios com adolescentes soropositivos na transição para a vida adulta $^{8}$, caracterizando tal fase como crítica para os portadores do vírus.

É curioso o fato de a amostra ao mesmo tempo apresentar médias altas para as dimensões do EMSV-A e médias altas para o fator depressão da EFN, sabendo-se que a dimensão satisfação de vida total correlaciona-se negativamente com esta última variável. A explicação possível para tal fato seria que as variáveis relacionadas às estratégias de coping ou de suporte social poderiam estar atuando como moderadoras entre o fator depressão e a satisfação de vida total, compreendendo-se variáveis moderadoras como aquelas que afetam a direção ou força da relação entre uma variável dependente e uma independente ${ }^{39}$. Ou seja, mesmo com características depressivas, jovens que possuem bom suporte social familiar (especialmente materno) e que conseguem lançar mão de um número variado de estratégias adequadas para lidar com os estressores atingem um bom nível de satisfação.

Esta pesquisa traz como contribuição um maior aprofundamento nas questões psicossociais, que não são tão exploradas em estudos com jovens portadores do vírus HIV. Através dos resultados, pode-se perceber onde residem as maiores dificuldades desse grupo e, dessa forma, elaborar programas de intervenção efetivos.

Percebe-se, porém, que pela possibilidade de existirem variáveis moderadoras atuando no resultado final da satisfação de vida, uma limitação deste estudo é o fato de a amostra ser composta de 45 participantes. Tal número é pequeno para a realização de análises como a de modelagem 
de equação estrutural, que são adequadas para a investigação de modelos com variáveis moderadoras. Sugere-se a realização de novas pesquisas que abarquem um número maior de participantes e que sejam avaliadas também outras variáveis que possam compor um modelo explicativo de satisfação de vida em jovens portadores do vírus.

\section{Referências}

1. ONUSIDA. Informe sobre la epidemia mundial de SIDA. Genebra: ONUSIDA; 2004

2. Brasil, Ministério da Saúde, Coordenação Nacional de DST e AIDS. Dados epidemiológicos AIDS. Bol Epidemiol AIDS e DST. 2007. http://www.aids.gov. br/data/Pages/LUMISD3352823PTBRIE.htm. Acessado 06 abr 2009.

3. Camargo BV, Botelho LJ. Aids, sexualidade e atitudes de adolescentes sobre proteção contra o HIV. Rev Saude Publica. 2007;41(1):61-8.

4. Oliveira SHS, Dias MR, Silva, MIT. Adolescentes e AIDS: fatores que influenciam a intenção de uso do preservativo. DST - J Bras Doenças Sex Transm. 2005;17(1):32-8.

5. Ruzany MH, Taquette SR, Oliveira RG, Meirelles ZV, Ricardo IB. A violência nas relações afetivas dificulta a prevenção de DST/AIDS? J Pediatr (Rio J) 2003;79(4):349-54.

6. Pechansky F, Bassols AMS, Diemen LV. Gênero, sexualidade e uso de drogas e adolescentes que realizaram o teste anti-HIV em um centro de testagem gratuita de Porto Alegre. Rev Psiquiatr RS. 2002;24(1):77-84

7. Taquette SR, Ruzany MH, Meirelles Z, Ricardo I. Relacionamento violento na adolescência e risco de DST/AIDS. Cad Saude Publica. 2003;19(5):1437-44.

8. Battles HB, Wiener LS. From adolescence through adulthood: psychosocial adjustment associated with long-term survival of HIV. J Adolesc Health. 2002;30(3):161-8.

9. Funck-Brentano I, Dalban C, Veber F, Quartier P, Hefez S, Costagliola D, et al. Evaluation of a peer support group therapy for HIV - infected adolescents. AIDS. 2005;19(14):1501-8.

10. Murphy DA, Moscicki AB, Vermund SH, Muenz LR. Psychological distress among HIV+ adolescents in the REACH study: effects of life stress, social support, and coping. The Adolescent Medicine HIV/AIDS Research Network. J Adolesc Health. 2000;27(6):391-8.

11. Abadía-Barrero CE, Castro A. Experiences of stigma and access to HAART in children and adolescents living with HIV/AIDS in Brazil. Soc Sci Med. 2006;62(5):1219-28.

12. Reppold CT, Reppold LT, Xavier ACM, Hutz CS. AIDS pediátrica: aspectos epidemiológicos, clínicos e socioemocionais da síndrome entre crianças e adolescentes infectados e suas famílias. Psico (Porto Alegre). 2004;35(1):79-88.

13. Seidl EMF, Rossi WS, Viana KF, Meneses AKF, Meireles E. Crianças e adolescentes vivendo com HIV/Aids e suas famílias: aspectos psicossociais e enfrentamento. Psicol Teor Pesqui. 2005;21(3):279-88.

14. Pavot W, Diener E, Colvin CR, Sandivik E. Further validation of the Satisfaction with Life Scale: evidence for the cross-method convergence of well-being measures. J Pers Assess. 1991;57(1):149-61.

15. Aldwin CM, Revenson TA. Does coping help? A reexamination of the relation between coping and mental health. J Pers Soc Psychol. 1987;53(2):337-48.

16. Diener E, Lucas R. Personality and subjective well-being. In: Kahneman D, Diener E, Schwarz N, org. Well-Being: the foundations of hedonic psychology. New York Russel Sage; 1999. p. 213-29.

17. Mccullough G, Huebner ES, Laughlin JE. Live events, self-concept, and adolescents'positive subjective well-being. Psychol Schools. 2000;37:281-90.
18. Nickerson AB, Nagle RJ. The influence of parent and peer attachments on life satisfaction in middle childhood and early adolescence. Soc Indic Res. 2004;66(12):35-60.

19. Canini SRMS, Reis RB, Pereira LA, Gir E, Pelá NTR. Qualidade de vida de indivíduos com HIV/AIDS: Uma revisão de literatura. Rev Latino-Am Enfermagem. 2004;12(6):940-5.

20. Leite OHM, Malbegier A, Santos CP, Nitrini R, Mathias SC, Nascimento VLV. Aspectos Clínicos e tratamentos específicos: manifestações psicológicas e psiquiátricas. In: Munhoz AL, Kieffer CR, Uip D, Oliveira MS, Leite OHM, org. HIV/AIDS: perguntas e respostas. São Paulo: Atheneu; 1996. p. 175-99.

21. Drotar DD, Angle DP, Eckl L, Thompson PA. Psychological response to HIV positivity in hemophilia. Pediatrics. 1995;96(6):1062-9.

22. Brown LK, Schultz JR, Gragg RA. HIV-infected adolescents with hemophilia: adaptation and coping. Pediatrics. 1995;96(3 Pt 1):459-63.

23. Heckman TG. The chronic illness quality of life (CIQOL) model: explaining life satisfaction in people living with HIV disease. Health Psychol. 2003;22(2):140-7.

24. Wiener L, Septimus A. Psychosocial consideration and support for child and family. In: Pizzo PA, Wilfred CM, org. Pediatric AIDS: the challenge of HIV infection in infants, children, and adolescents. Baltimore: Williams \& Wilkins; 1991. p. 577-94.

25. Lazarus R, Folkman S. Stress, appraisal and coping. New York: Springer; 1984.

26. Meijer SA, Sinnema G, Bijstra JO, Mellenbergh GJ, Wolters WH. Coping styles and locus of control as predictors for psychological adjustment of adolescents with a chronic illness. Soc Sci Med. 2002;54(9):1453-61.

27. Associação Brasileira de Empresas de Pesquisa. Critério de classificação econômica Brasil da associação brasileira de empresas de pesquisa. http://www. abep.org/codigosguias/ABEP_CCEB_2003.pdf. Acessado 10 Dez 2006.

28. Arteche AX. O Impacto do trabalho nas variáveis coping e bem-estar subjetivo em uma amostra de adolescentes [dissertação]. Porto Alegre: UFRGS; 2003.

29. Giacomoni CH, Hutz CS. Escala multidimensional de satisfação de vida para crianças: estudos de construção e validação. Estud Psicol (Campinas). 2008;25(1):23-35.

30. Furman W, Buhrmester D. Age and sex differences in perceptions of networks of personal relationships. Child Dev. 1992;63(1):103-15.

31. Marques JC, Van Horn KR. Relações interpessoais em pré-adolescentes, adolescentes e universitários brasileiros: um estudo transcultural. Psico (Porto Alegre). 2002;33(2):245-72.

32. Folkman S, Lazarus RS. If it changes it must be a process: study of emotion and coping during three stages of a college examination. J Pers Soc Psychol. 1985;48(1):150-70.

33. Savóia MG, Santana PR, Mejias NP. Adaptação do inventário de estratégias de coping de Folkman e Lazarus para o português. Psicol USP. 1996;7(1-2):183-201.

34. Hutz CS, Nunes CHS. Escala fatorial de Ajustamento Emocional/ Neuroticismo - EFN. São Paulo: Casa do Psicólogo; 2001.

35. Furman W, Buhrmester D. Childrens perceptions of the personal relationships in their social networks. Develop Psychol. 1985;21(6):1016-24.

36. Cordeiro RA. Aparência física e amizade íntima na adolescência: estudo num contexto pré-universitário. Ana Psicológica. 2006;24(4):509-17.

37. Taquette SR, Vilhena MM, Paula MC. Doenças sexualmente transmissíveis e gênero: um estudo transversal com adolescentes no Rio de Janeiro. Cad Saude Publica. 2004;20(1):282-90.

38. Vieira MAS, Guimarães EMB, Barbosa MA, Turchi MD, Alves MFC, Seixas MSC, et al. Fatores associados ao uso de preservativo em adolescentes do gênero feminino no município de Goiânia. DST J Bras Doenças Sex Transm. 2004;16(3):77-83.

39. Baron RM, Kenny D. The moderator-mediator variable distinction in socia psychological research: conceptual, strategic, and statistical considerations. J Pers Soc Psychol. 1986;51(6):1173-82. 